\title{
New Multiscale Models and Self-Similarity in Tropical Convection
}

\author{
ANDREW J. MAJDA \\ Department of Mathematics, and Center for Atmosphere-Ocean Science, Courant Institute, New York University,
} New York, New York

(Manuscript received 14 April 2006, in final form 17 July 2006)

\begin{abstract}
One of the unexplained striking features of tropical convection is the observed statistical self-similarity in clusters, superclusters, and intraseasonal oscillations through complex multiscale processes ranging from the mesoscales to the equatorial synoptic scales to the intraseasonal/planetary scales. Here new multispatialscale, multitime-scale, simplified asymptotic models are derived systematically from the equatorial primitive equations on the range of scales from mesoscale to equatorial synoptic to planetary/intraseasonal, which provide a useful analytic framework for addressing these issues. New mesoscale equatorial synoptic dynamical (MESD) models and balanced MESD (BMESD) models are developed for the multitime, multispace interaction from mesoscales to equatorial synoptic scales; new multitime versions of the intraseasonal planetary equatorial synoptic dynamics (IPESD) models are developed for multiple spatiotemporal interactions on equatorial synoptic scales and planetary scales. The mathematical character derived below for all these simplified models explicitly demonstrates that the main nonlinear interactions across scales are quasi-linear where eddy flux divergences of momentum and temperature from nonlinear advection from the smaller-scale spatiotemporal flows as well as mean source effects accumulate in time and drive the waves on the successively larger spatiotemporal scales. Furthermore, these processes that transfer energy to the next larger, longer, spatiotemporal scales are self-similar in a suitable sense established here. On the other hand, the larger scales set the environment for this transport through processes such as mean advection of the smaller scales.
\end{abstract}

\section{Introduction}

Observational data indicate that through the complex interaction of heating and convection, tropical atmospheric flows are organized on a hierarchy of scales (Nakazawa 1988) ranging from cumulus clouds of a few kilometers to mesoscale convective systems (Houze 2004) to equatorial synoptic-scale convectively coupled Kelvin waves and 2-day waves (Wheeler and Kiladis 1999) to planetary-scale intraseasonal organized circulations such as the Madden-Julian oscillation (MJO; Hendon and Salby 1994). Futhermore, recent processing of observational data (Mapes et al. 2006) suggests the statistical self-similarity of tropical convection on multiple spatiotemporal scales ranging from the mesoscales to the planetary scales. On the other hand, contemporary general circulation models (GCMs) perform

Corresponding author address: Dr. Andrew J. Majda, Courant Institute, New York University, 251 Mercer St., New York, NY 10012.

E-mail: jonjon@cims.nyu.edu poorly both in their ability to resolve convectively coupled synoptic-scale waves and the MJO (Slingo et al. 1996; Lin et al. 2006). One conjectured reason for this inadequate performance of contemporary GCMs is their inability to represent adequately the statistical interaction of moist convection across multiple spatiotemporal scales (Moncrieff and Klinker 1997; Moncrieff 2004). For all the above reasons, it is interesting to develop systematic multiscale asymptotic models (Klein 2000; Majda 2003; Majda and Klein 2003) including spatiotemporal scales spanning the mesoscales to the equatorial synoptic scales to the planetary/intraseasonal scales. This is the topic of the present paper.

For a wide variety of potential applications ranging from the development of equatorial synoptic-scale superclusters with embedded convective mesoscale clusters as well as tropical cyclogenesis, it is interesting to develop systematic simplified multiscale models from the equatorial primitive equations that incorporate multiple space/time-scale interactions from the equatorial synoptic scale, $O(1500 \mathrm{~km})$, and the mesoscale, $O(150 \mathrm{~km})$, on both the equatorial synoptic time scale

DOI: $10.1175 / \mathrm{JAS} 3880.1$

(C) 2007 American Meteorological Society 
$O(8 \mathrm{~h})$ and the mesoscale time $O(1 \mathrm{~h})$. For example, organized synoptic-scale convectively coupled eastward-propagating Kelvin waves and westwardpropagating 2-day waves with propagation speeds of roughly $15 \mathrm{~m} \mathrm{~s}^{-1}$ arise as the propagating envelope of a complex multiscale environment involving organized mesoscale convective systems (MCSs). Current theories that parameterize key features of the MCSs are capable of reproducing many observational features of these equatorial synoptic-scale waves, such as their phase speed and vertical tilt, as a nonlinear convectively coupled response on the synoptic scales (Mapes 2000; Majda and Shefter 2001; Majda et al. 2004; Khouider and Majda 2006, 2007). However, it is important to understand how much of the synoptic-scale response is due simply to the induced mean heating on the synoptic scale versus the upscale transports of momentum and temperature from the mesoscale convective wave trains and, furthermore, how the synoptic scale flows alter the organized mesoscale wave trains to create such propagation. In particular, Haertel and Kiladis (2004) show for 2-day waves that the dynamics of the linear equatorial primitive equations with the total imposed multiscale heating from the observations is sufficient to capture many salient features of these waves. The simplified multiscale asymptotic models derived in detail below in section 3 , the mesoscale equatorial synoptic dynamics (MESD) and balanced MESD (BMESD), provide a diagnostic framework to specifically address the above issues in a quantitative fashion either in observations (Wheeler and Kiladis 1999; Haertel and Kiladis 2004) or in cloud-resolving numerical models (Grabowski and Moncrieff 2001; Tulich et al. 2007).

Simplified balanced models for the interaction of equatorial synoptic-scale, $O(1000-1500 \mathrm{~km})$, wave patterns with the planetary-scale circulations $O(10000$ $\mathrm{km}$ ) on intraseasonal time scales $O(30$ days) have been introduced recently (Majda and Klein 2003). These are the Intraseasonal Planetary Equatorial Synoptic Dynamics (IPESD) models. The IPESD models have been utilized to give insight into the detailed multiscale structure of the MJO in a fashion consistent with the observational record including the important issues of the relative role of planetary-scale heating compared with upscale eddy flux convergence of momentum and temperature in determining the structure of the MJO (Majda and Biello 2004; Biello and Majda 2005, 2006a,b). The original IPESD models allowed for only a single intraseasonal time scale and the spatial interaction from the equatorial synoptic to the planetary scales. Here the IPESD models are systematically generalized to allow for multiple time scales involving fluctuating behavior on the equatorial synoptic time scale $O(8 \mathrm{~h})$ in addition to variations on the intraseasonal time scale as well as interaction across the multiple spatial scales. The simplicity of this reduced asymptotic multitime IPESD model as well as its structural similarity with the multiple spatiotemporal MESD models for the interaction of mesoscale and equatorial synoptic scale waves are crucial for the perspective on statistical self-similarity of tropical convection developed below. In the original IPESD models, the phase speed of the MJO needs to be specified; the same remarks apply to the MESD and multiscale IPESD models in the present paper. The nonlinear interactive effects of active moist processes need to be included in these models in order to actually predict such phase speeds (Khouider and Majda 2006).

In this paper, new multiple space-scale and single or multiple time-scale simplified asymptotic models are developed both for the interaction of mesoscale disturbances with equatorial synoptic-scale waves on the equatorial synoptic time scale and for the interaction of equatorial synoptic-scale waves and planetary-scale circulations on the intraseasonal time scale. In the first situation, the basic two length scales are the equatorial synoptic scale, $L_{E}=L_{S}$, and the mesoscale $L_{M}$ :

$$
\begin{aligned}
L_{S} & =L_{E} \approx O(1500 \mathrm{~km}), \\
L_{M} & =\epsilon L_{S} \approx O(150-200 \mathrm{~km}),
\end{aligned}
$$

with synoptic and mesoscale time scales:

$$
T_{S}=T_{E} \approx O(8 \mathrm{hrs}), \quad T_{M}=\epsilon T_{S} \approx O(1 \mathrm{hr}) .
$$

For the multiscale interactions for equatorial synoptic and planetary scales, the basic length and time scales are equatorial synoptic and planetary/intraseasonal (Majda and Klein 2003):

$$
\begin{aligned}
& L_{S}=L_{E} \approx O(1500 \mathrm{~km}), \\
& L_{P}=\epsilon^{-1} L_{S} \approx O(10000 \mathrm{~km}), \\
& T_{S}=T_{E} \approx O(8 \mathrm{hrs}), \quad T_{I}=\epsilon^{-1} T_{S} \approx O(3 \text { days }) .
\end{aligned}
$$

See Fig. 1. Note that the multiple scales in (1) and (2) span all the relevant spatiotemporal scales in the Tropics from mesoscale to planetary/intraseasonal with the equatorial synoptic scales as the crucial overlap scales between those in (1) and (2); as discussed earlier (Majda and Klein 2003), with conservative realistic values of $\epsilon \approx 0.1$, the asymptotic models developed below retain their validity for roughly 10 time units of the longest time scale, roughly 1 month for (2), that is, intraseasonal time scales, and roughly $3-4$ days for the interactions in (1), that is, synoptic time scales. New simplified multiscale balanced models for the situation in (1), called MESD and BMESD, are developed in section 3 


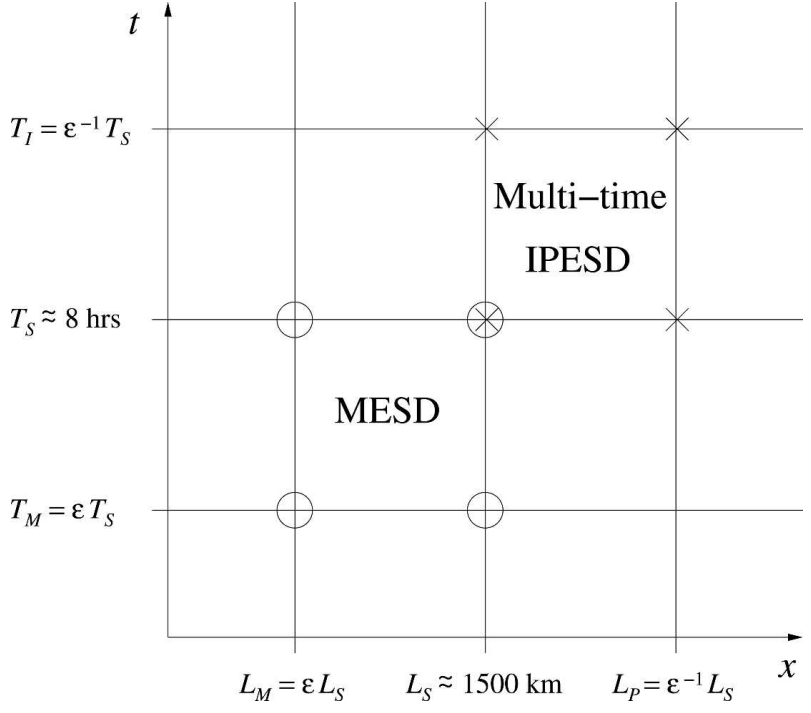

FIG. 1. The multiple spatiotemporal scales spanned here with the MESD and multitime IPESD models with overlap on equatorial synoptic scales.

below while new multitime IPESD models are developed in section 4 below for the situation in (2). In section 5, the self-similar scaling structure within these simplified asymptotic models across all the spatiotemporal scales in (1) and (2) is utilized to put forward a quantitative framework for the statistical self-similarity of tropical convection from mesoscales to planetary scales on both tropical weather and intraseasonal time scales. In essence, the theory developed in section 5 establishes that as regards the zonal direction of propagation, the nonlinear dynamics in the MESD models operating on the smaller, shorter spatiotemporal scales in (1) is exactly the same as the multitime IPESD dynamics acting on the longer spatiotemporal scales in (2). This is achieved through a simple self-similar rescaling transformation relating (1) and (2). Furthermore, this interlocking and overlap of spatiotemporal scales is satisfied self-consistently through a single asymptotic parameter $\epsilon$ defined in (4) below which sets the low Froude number and weak temperature gradient amplitudes.

The development of systematic balanced models involving a single spatiotemporal scale has a long tradition for geophysical flows beginning with quasigeostrophic dynamics for midlatitudes, where there is strict temporal-scale separation due to the nonvanishing of Coriolis forces. In this single-scale midlatitude context, one can usually apply simple scaling analysis with estimates of orders of magnitude for each term to derive the simplified quasigeostrophic model or alternatively utilize a more concise asymptotic expansion to derive the equations (Pedlosky 1987; Majda 2003). In the Tropics, the effect of rotation degenerates at the equator and interesting flows often have substantial strong forcing from convection so the systematic asymptotic procedures are useful for deriving single-scale balanced models for the Tropics (Majda and Klein 2003; Majda 2003). It is much more challenging to develop systematic simplified models for complex systems such as the primitive equations when physical phenomena vary on multiple spatial and temporal scales simultaneously and one cannot guess the form of the equations by simple scale analysis. Here one needs to utilize the tools of systematic multiscale perturbation theory from applied mathematics to understand this coupling (Kevorkian and Cole 1981; Embid and Majda 1998) where the crux of these arguments involves eliminating higher-order secular terms in an asymptotic expansion (Embid and Majda 1998); for complex partial differential equations, this is often a subtle mathematical task (see, e.g., Klein 2000; Majda 2003; Majda and Klein 2003). On the other hand, it is precisely these constraints imposed by eliminating suitable secular terms, which lead to the simplified reduced equations. The new technical challenge in the present paper is to utilize these systematic mathematical multiscale procedures to develop simplified equations for the rather complex situations described above involving interactions across multiple spatiotemporal scales in the equatorial waveguide with strong fluctuating forcing. Thus, some of the mathematical developments, by necessity, are quite involved.

\section{Preliminaries}

The systematic multiscale models developed in the subsequent sections are derived systematically from the anelastic primitive equations on the equatorial $\beta$ plane. In standard nondimensional units, these equations are given by

$$
\begin{aligned}
\frac{D}{D t} u-y v & =-p_{x}+S_{u}, \\
\frac{D}{D t} v+y u & =-p_{y}+S_{v}, \\
\frac{D}{D t} \theta+N^{2} w & =S_{\theta}, \\
p_{z} & =\theta, \\
(\rho u)_{x}+(\rho v)_{y}+(\rho w)_{z} & =0,
\end{aligned}
$$

where $\rho=\rho(z)$ and $N^{2}=N^{2}(z)$ are the density and buoyancy frequency, respectively, and depend on the height in the troposphere alone. The time scales, $t$, are nondimensionalized with the unit of the equatorial time 
scale, $T_{E}=(c \beta)^{-1 / 2} \approx 8.3 \mathrm{~h}$, the horizontal length scales to the equatorial deformation radius, $L_{E}=(c / \beta)^{1 / 2} \approx$ $1500 \mathrm{~km}$, and the vertical length scale to the troposphere height divided by $\pi, H_{T} / \pi \approx 5 \mathrm{~km}$, so that $H_{T}=$ $16 \mathrm{~km}$. The horizontal velocities are scaled to the dry Kelvin-gravity wave speed, $c=50 \mathrm{~m} \mathrm{~s}^{-1}$ whereas the vertical velocity is scaled to the vertical/horizontal aspect ratio times this speed, $=5 / 1500 c \approx 0.16 \mathrm{~m} \mathrm{~s}^{-1}$. The temperature scale is equal to the thermal lapse rate in the atmosphere measured over one unit of the vertical scale, $[\theta] \approx 33 \mathrm{~K}$. In these units, the density is scaled to that at the base of the free troposphere, $\rho(z=0)=1$ (and is elsewhere less than or equal to 1) and the buoyancy frequency (whose average in the troposphere is about $10^{-2} \mathrm{~s}^{-1}$ ) is equal to 1 plus a small variation as a function of height, $N^{2}(z)=1+\mu(z)$. In this nondimensionalization, the free troposphere occupies the domain $0 \leq z \leq \pi$, with a rigid lid at the top of the troposphere, that is to say that the vertical velocity vanishes there and at $z=0$. The advective nonlinearity in (3) is given by

$$
\frac{D}{D t}=\frac{\partial}{\partial t}+u \frac{\partial}{\partial x}+v \frac{\partial}{\partial y}+w \frac{\partial}{\partial z} .
$$

It is useful to remark here that by using the conservation of mass equation from (3e), the horizontal momentum Eqs. (3a)-(3b) can be put in the conservation form

$$
\frac{\partial}{\partial t}\left(\rho \mathbf{u}_{h}\right)+\operatorname{div}\left(\rho \mathbf{u}: \mathbf{u}_{h}\right)+\rho y \mathbf{u}_{h}^{\perp}=-\nabla_{h}(\rho p)+\rho \mathbf{S}_{\mathbf{u}},
$$

with $\mathbf{x}=(x, y), \mathbf{u}_{h}=(u, v), \mathbf{u}_{h}^{\perp}=(-v, u), \nabla_{h}=(\partial / \partial x$, $\partial / \partial y)$, and $\left(\rho \mathbf{u}: \mathbf{u}_{h}\right)_{i}=\rho \mathbf{u u}_{h, i}, i=1,2$.

Systematic multiscale asymptotic solutions of (3) involving all the spatiotemporal scales in (1) and (2) are developed with the following basic assumptions:

Assumption 1: Low Froude number, $\mathbf{u}_{h}=\boldsymbol{\epsilon} \mathbf{u}_{h, 1}$

Assumption 2: Weak temperature gradient, $\theta=\epsilon \theta_{1}$,

$$
p=\epsilon p_{1},
$$

as well as the equatorial $\beta$-plane approximation tacit in (3). The strength and structure of the heat and momentum sources for the synoptic-planetary-scale interactions on intraseasonal time scales is already familiar (Majda and Klein 2003; Majda and Biello 2004; Biello and Majda 2005, 2006a,b) and will be summarized at the beginning of section 4 below. In general below a subscript is utilized to coincide with the multiplying power in $\epsilon$ as in (4); however, this notation is abused slightly for example, in (8), for the terms of leading order to avoid cumbersome notation in the final derived equations. In the remainder of this preliminary section, this structure is discussed for the mesoscale/

\begin{tabular}{|c|c|c|}
\hline Variable & Resolved scales & Description \\
\hline $\mathbf{x}=(x, y)$ & $O(1500 \mathrm{~km})$ & Equatorial synoptic scale \\
\hline$t$ & $O(8 \mathrm{~h})$ & $\begin{array}{l}\text { Equatorial synoptic time } \\
\text { scale }\end{array}$ \\
\hline$x^{\prime}=\epsilon^{-1} x$ & $O(150 \mathrm{~km})$ & Mesoscale \\
\hline$\tau=\epsilon^{-1} t$ & $O(1 \mathrm{~h})$ & Mesoscale time \\
\hline$X=\epsilon x$ & $O(10000 \mathrm{~km})$ & Zonal planetary scale \\
\hline$T=\epsilon t$ & $O(3.5$ days $)$ & Intraseasonal time scale \\
\hline $\bar{f}$ & \multicolumn{2}{|c|}{ Spatial average over a given smaller scale } \\
\hline$f^{\prime}$ & \multicolumn{2}{|c|}{$\begin{array}{l}\text { Spatial fluctuation over a given smaller scale with } \\
\bar{f}^{\prime}=0\end{array}$} \\
\hline$\langle f\rangle$ & \multicolumn{2}{|c|}{ Temporal average over a given smaller scale } \\
\hline
\end{tabular}
equatorial synoptic-scale regime in (1).
TABLE 1. Basic units and notation.

\section{Multiple mesoscale and equatorial synoptic scales}

For the situation in (1), the temperature and momentum sources for (3) are assumed to involve the multiple scales in (1), that is, $S_{\theta}\left(\mathbf{x}, \epsilon^{-1} \mathbf{x}, z, t, \epsilon^{-1} t\right), \mathbf{S}_{\mathbf{u}}\left(\mathbf{x}, \epsilon^{-1} \mathbf{x}, z\right.$, $\left.t, \epsilon^{-1} t\right)$, where the notation $\mathbf{x}^{\prime}=\epsilon^{-1} \mathbf{x}$ will be utilized below. Note that given $f\left(\mathbf{x}, \epsilon^{-1} \mathbf{x}\right)$ the total horizontal gradient of $f$ is given by

$$
\boldsymbol{\nabla}_{h} f+\epsilon^{-1} \nabla_{h}^{\prime} f,
$$

where in (5) and below, $\nabla_{h}$ denotes the gradient with respect to the large-scale variables while $\boldsymbol{\nabla}_{h}^{\prime}$ denotes the gradient with respect to $\mathbf{x}^{\prime}$. Given $f\left(\mathbf{x}, \epsilon^{-1} \mathbf{x}\right)$, the largescale average of $f$ is given by

$$
\bar{f}(\mathbf{x})=\lim _{L \rightarrow \infty}(2 L)^{-2} \int_{-L}^{L} \int_{-L}^{L} f\left(\mathbf{x}, \mathbf{x}^{\prime}\right) d x^{\prime} d y^{\prime},
$$

and $f\left(\mathbf{x}, \mathbf{x}^{\prime}\right)=\bar{f}(\mathbf{x})+f^{\prime}\left(\mathbf{x}, \mathbf{x}^{\prime}\right)$ with $\overline{f^{\prime}}=0$; below, the notation $f^{\prime}$ always denotes a variable with vanishing large-scale average $\left(\overline{f^{\prime}}=0\right)$. These and the other basic asymptotic space-time units and notation for this paper are summarized in Table 1. With these preliminaries, the source terms $S_{\theta}, \mathbf{S}_{\mathbf{u}}$ satisfy the following assumptions:

$$
\begin{aligned}
& S_{\theta}\left(\mathbf{x}, \mathbf{x}^{\prime}, z, t, \epsilon^{-1} t\right)=S_{\theta}^{\prime}+\epsilon S_{\theta, 1}, \\
& \mathbf{S}_{\mathbf{u}}\left(\mathbf{x}, \mathbf{x}^{\prime}, z, t, \epsilon^{-1} t\right)=\mathbf{S}_{\mathbf{u}}^{\prime}+\epsilon \mathbf{S}_{\mathbf{u}, 1} .
\end{aligned}
$$

These assumptions on the strength of the sources needed in (7) are satisfied by the typical observed heat sources in the Tropics on both mesoscales and equatorial synoptic scales. Note that with the standard nondimensionalization discussed after (3), if $S_{\theta}^{\prime} \neq 0$, then there are strong fluctuating heat sources on the mesoscales with strength roughly $30 \mathrm{~K}(8 \mathrm{~h})^{-1}$ while the magnitude of $S_{\theta, 1}$ corresponds to weaker heat sources of roughly $10 \mathrm{~K} \mathrm{day}^{-1}$ for $\epsilon \approx 0.1$. Similarly, the momentum source $\epsilon \mathbf{S}_{\mathbf{u}, 1}$ has strength up to roughly 15 $\mathrm{m} \mathrm{s}^{-1}$ day $^{-1}$. Note that any fluctuations $\mathbf{S}_{\mathbf{u}}^{\prime}, S_{\theta}^{\prime}$ that on the mesoscale induce vertical velocity $w^{\prime}$ of roughly 
$0.2 \mathrm{~m} \mathrm{~s}^{-1}$ are compatible with these strong variations imposed here according to the nondimensionalization after (3).

\section{Simplified equations for mesoscale-equatorial synoptic-scale interaction}

Under the assumptions of low Froude number, weak temperature gradient, and fluctuating heat sources developed in (4) and (7), there is a systematic asymptotic expansion of the primitive equations on equatorial synoptic time scales with the following form:

$$
\begin{aligned}
\mathbf{u}_{h} & =\epsilon \overline{\mathbf{u}}\left(\mathbf{x}, z, t, \epsilon^{-1} t\right)+\epsilon \mathbf{u}_{h}^{\prime}\left(\mathbf{x}, \epsilon^{-1} \mathbf{x}, z, t, \epsilon^{-1} t\right)+\epsilon^{2} \mathbf{u}_{h, 2}, \\
w & =w^{\prime}+\epsilon \bar{w}+\epsilon w_{1}^{\prime}+O\left(\epsilon^{2}\right), \\
\theta & =\epsilon \bar{\theta}+\epsilon \theta^{\prime}+O\left(\epsilon^{2}\right), \\
p & =\epsilon \bar{p}+\epsilon p^{\prime}+O\left(\epsilon^{2}\right),
\end{aligned}
$$

where $\mathbf{x}$ describes the spatial scale of variation on the equatorial synoptic scale and $\mathbf{x}^{\prime}=\epsilon^{-1} \mathbf{x}$ denotes the spatial scale of variation on the mesoscales while $\mathbf{u}_{h}=$ $(u, v)$ is the horizontal velocity. The variable $\tau=\epsilon^{-1} t$ denotes the time scale for temporal fluctuations on the shorter mesoscale time. As explained in (6) above, $\bar{f}$ denotes the synoptic-scale average while $f^{\prime}$ denotes the mesoscale fluctuations part with $\bar{f}^{\prime}=0$.

The multispatial-scale BMESD suppresses temporal fluctuations on the mesoscale so that the dependence in (8) on $\epsilon^{-1} t$ is ignored. The BMESD model, which is systematically derived in detail below, is given by the following two pieces: equations for the balanced mesoscale fluctuations including propagation and generation of vorticity as well as equatorial anelastic linear equations for the synoptic-scale flow; they are nonlinearly coupled through eddy flux divergence, large-scale advection of fluctuating vorticity, and mesoscale vorticity production through the large scales. Given the decomposition in (8), here are the BMESD equations:

1) Equatorial synoptic-scale dynamics:

$$
\begin{aligned}
\frac{\partial}{\partial t}\left(\rho \overline{\mathbf{u}}_{h}\right)+\rho y \overline{\mathbf{u}}_{h}^{\perp} & =-\nabla_{h}(\rho \bar{p})-\left(\rho \overline{w^{\prime} \mathbf{u}_{h}^{\prime}}\right)_{z}+\rho \overline{\mathbf{S}}_{\mathbf{u}, 1} \\
\frac{\partial}{\partial t}(\rho \bar{\theta})+N^{2} \rho \bar{w} & =-\left(\rho \overline{w^{\prime} \theta^{\prime}}\right)_{z}+\rho \bar{S}_{\theta, 1} \\
\bar{p}_{z} & =\bar{\theta} \\
\operatorname{div}_{h} \overline{\mathbf{u}}_{h} & =-\frac{1}{\rho}(\rho \bar{w})_{z}
\end{aligned}
$$

coupled with

2) Balanced mesoscale fluctuation dynamics with $\Phi_{s}^{\prime}$ as the velocity potential for the momentum sources,

$$
p^{\prime}=\Phi_{s}^{\prime}, \quad \theta^{\prime}=p_{z}^{\prime}, \quad N^{2}(z) w^{\prime}=S_{\theta}^{\prime}
$$

and with the Helmholtz decomposition on mesoscales, $\mathbf{u}_{h}^{\prime}=\nabla_{h}^{\prime} \Phi^{\prime}+\nabla_{h}^{\prime \perp} \Psi^{\prime}$

$$
\operatorname{div}_{h}^{\prime} \mathbf{u}_{h}^{\prime}=\Delta_{h}^{\prime} \Phi^{\prime}=-\frac{1}{\rho}\left(\rho w^{\prime}\right)_{z} .
$$

The mesoscale fluctuating vertical vorticity $\Delta_{h}^{\prime} \Psi^{\prime}=$ $\omega^{\prime}$ satisfies

$$
\begin{aligned}
\frac{\partial \omega^{\prime}}{\partial t} & +\overline{\mathbf{u}}_{h} \cdot \nabla_{h}^{\prime} \omega^{\prime}+\mathbf{u}_{h}^{\prime} \cdot \nabla_{h}^{\prime} \omega^{\prime}+w^{\prime} \frac{\partial \omega^{\prime}}{\partial z}+\left(\omega^{\prime}+y\right) \\
& \times\left[-\frac{1}{\rho}\left(\rho w^{\prime}\right)_{z}\right]+\left(\nabla_{h}^{\prime} w^{\prime}\right) \cdot \frac{\partial \mathbf{u}_{h}}{\partial z} \\
& =-\operatorname{curl}^{\prime}\left(\nabla_{h} \Phi_{s}^{\prime}\right)+\operatorname{curl}^{\prime} \mathbf{S}_{\mathbf{u}, 1}^{\prime} .
\end{aligned}
$$

The simplified MESD model derived below involves all the multiple spatiotemporal scales in (1). The derivation begins with the general ansatz in (8). As necessary background to describe the MESD model, for a general function $f\left(\mathbf{x}, \epsilon^{-1} \mathbf{x}, z, t, \epsilon^{-1} t\right)$, the average over the mesoscale temporal fluctuations is defined by

$$
\langle f\rangle\left(\mathbf{x}, \epsilon^{-1} \mathbf{x}, z, t\right)=\lim _{T \rightarrow \infty} \frac{1}{2 T} \int_{-T}^{T} f\left(\mathbf{x}, \epsilon^{-1} \mathbf{x}, z, t, \tau\right) d \tau .
$$

In the MESD models, the leading-order mesoscale fluctuations involve forced linear anelastic waves on the mesoscale time that supply eddy flux divergence for momentum and temperature for the equatorial anelastic equations on synoptic space and time scales. With the nonresonant forcing hypothesis for $\mathbf{S}_{\mathbf{u}}^{\prime}, S_{\theta}^{\prime}$ and derivation below, here are the MESD equations:

1) Equatorial synoptic-scale dynamics: $\left\langle\overline{\mathbf{u}}_{h}\right\rangle,\langle\bar{\theta}\rangle,\langle\bar{p}\rangle$, $\langle\bar{w}\rangle(\mathbf{x}, z, t)$ satisfy

$$
\begin{aligned}
\frac{\partial}{\partial t}\left(\rho\left\langle\overline{\mathbf{u}}_{h}\right\rangle\right)+\rho y\left\langle\overline{\mathbf{u}}_{h}\right\rangle^{\perp}= & -\nabla_{h}(\rho\langle\bar{p}\rangle)-\left(\rho\left\langle\overline{w^{\prime} \mathbf{u}_{h}^{\prime}}\right\rangle\right)_{z} \\
& +\rho\left\langle\overline{\mathbf{S}}_{\mathbf{u}, 1}\right\rangle, \\
\frac{\partial}{\partial t}(\rho\langle\bar{\theta}\rangle)+N^{2} \rho\langle\bar{w}\rangle= & -\left(\rho\left\langle\overline{w^{\prime} \theta^{\prime}}\right\rangle\right)_{z}+\rho\left\langle\bar{S}_{\theta, 1}\right\rangle
\end{aligned}
$$

$$
\begin{aligned}
\langle\bar{p}\rangle_{z} & =\langle\bar{\theta}\rangle, \\
\operatorname{div}_{h}\left\langle\overline{\mathbf{u}}_{h}\right\rangle & =-\frac{1}{\rho}(\rho\langle\bar{w}\rangle)_{z},
\end{aligned}
$$

while the mesoscale spatiotemporal fluctuations satisfy the 
2) Forced linear anelastic equations:

$$
\begin{aligned}
\frac{\partial \mathbf{u}_{h}^{\prime}}{\partial \tau} & =-\nabla_{h}^{\prime} p^{\prime}+\mathbf{S}_{\mathbf{u}}^{\prime}\left(\mathbf{x}, \mathbf{x}^{\prime}, z, t, \tau\right), \\
\frac{\partial \theta^{\prime}}{\partial \tau}+N^{2} w^{\prime} & =S_{\theta}^{\prime}\left(\mathbf{x}, \mathbf{x}^{\prime}, z, t, \tau\right), \\
p_{z}^{\prime} & =\theta^{\prime}, \\
\operatorname{div}_{h}^{\prime} \mathbf{u}_{h}^{\prime}+\frac{1}{\rho}\left(\rho w^{\prime}\right)_{z} & =0 .
\end{aligned}
$$

The MESD equations are a quasi-linear model. Given the structure of the forcings $\mathbf{S}_{\mathbf{u}}^{\prime}, S_{\theta}^{\prime}$ explicit solutions of the linear equations in (13) can be found that can be utilized explicitly to determine the eddy fluxes $\left\langle\overline{\left\langle w^{\prime} \mathbf{u}_{h}^{\prime}\right.}\right\rangle$, $\left\langle\overline{\left\langle w^{\prime} \theta^{\prime}\right.}\right\rangle$, which drive the linear equatorial synoptic-scale equations in (12). Thus, the analytic structure is similar but with different physical mechanisms to the multiscale IPESD models for planetary-scale and equatorial synoptic-scale interaction (Majda and Klein 2003); such models are useful for linking multiscale features with observations for the MJO (Majda and Biello 2004; Biello and Majda 2005, 2006a,b). One can anticipate a similar role for the MESD and BMESD models in sorting out the cluster-supercluster interactions. The equations in (13) need to be solved on the longer time scale in order to compute the required eddy fluxes (Majda 2003); the details will be presented elsewhere in a detailed application.

\section{a. The asymptotic derivation of BMESD}

With the assumptions in (4) and (7), and ignoring the temporal mesoscale fluctuations involving $\tau=\epsilon^{-1} t$, it is natural to seek multiple spatial-scale single time-scale asymptotic solutions of (3) with the following form:

$$
\begin{aligned}
\mathbf{u}_{h} & =\epsilon \overline{\mathbf{u}}_{h}(\mathbf{x}, z, t)+\epsilon \mathbf{u}_{h}^{\prime}\left(\mathbf{x}, \epsilon^{-1} \mathbf{x}, z, t\right)+\epsilon^{2} \mathbf{u}_{h, 2}^{\prime}, \\
w & =w^{\prime}+\epsilon \bar{w}+\epsilon w_{1}^{\prime}+O\left(\epsilon^{2}\right), \\
\theta & =\epsilon \bar{\theta}+\epsilon \theta^{\prime}+\epsilon^{2} \theta_{2}, \\
p & =\epsilon \bar{p}+\epsilon p^{\prime}+\epsilon^{2} p_{2} .
\end{aligned}
$$

First, the hydrostatic balance in (3) yields at leading order, $p_{z}^{\prime}=\theta^{\prime}, \bar{p}_{z}=\bar{\theta}$. Substituting the ansatz from (14) into the remaining equatorial primitive equations in (3) yields the following equations so that the terms of bounded order [i.e., $O\left(\epsilon^{0}\right)$ ] vanish:

$$
\begin{aligned}
\nabla_{h}^{\prime} p^{\prime} & =\mathbf{S}_{\mathbf{u}}^{\prime}, \\
N^{2} w^{\prime} & =S_{\theta}^{\prime}, \\
\operatorname{div}_{h}^{\prime} \mathbf{u}_{h}^{\prime} & =-\frac{1}{\rho}\left(\rho w^{\prime}\right)_{z}, \\
p_{z}^{\prime} & =\theta^{\prime},
\end{aligned}
$$

and also $O(\epsilon)$ :

$$
\begin{aligned}
& \frac{\partial}{\partial t}\left(\rho \mathbf{u}_{h}\right)+\operatorname{div}_{h}^{\prime}\left(\rho \mathbf{u}_{h}: \mathbf{u}_{h}\right)+\left(\rho w^{\prime} \mathbf{u}_{h}\right)_{z}+y \rho \mathbf{u}_{h}^{\perp} \\
& =-\nabla_{h}(\rho p)-\nabla_{h}^{\prime}\left(\rho p_{2}^{\prime}\right)+\rho \mathbf{S}_{\mathbf{u}, 1} \\
& \frac{\partial}{\partial t}(\rho \theta)+\operatorname{div}_{h}^{\prime}\left(\rho \mathbf{u}_{h} \theta\right)+\left(\rho w^{\prime} \theta\right)_{z}+N^{2} \rho w_{1}=\rho S_{\theta, 1}, \\
& \operatorname{div}_{h} \mathbf{u}_{h}+\operatorname{div}_{h}^{\prime} \mathbf{u}_{h, 2}^{\prime}=-\frac{1}{\rho}\left(\rho w_{1}\right)_{z}
\end{aligned}
$$

with $\mathbf{u}_{h}=\overline{\mathbf{u}}_{h}+\mathbf{u}_{h}^{\prime}, w_{1}=\bar{w}+w_{1}^{\prime}, \theta=\bar{\theta}+\theta^{\prime}, p=\bar{p}+$ $p^{\prime}$. Introduce the two-dimensional Helmholtz decomposition with the vertical coordinate as a parameter, $\mathbf{u}_{h}^{\prime}=\nabla_{h}^{\prime} \Phi^{\prime}+\left(\nabla_{h}^{\prime}\right)^{\perp} \Psi^{\prime}$, and assume that the momentum source $\mathbf{S}_{\mathbf{u}}^{\prime}$ satisfies $\mathbf{S}_{\mathbf{u}}^{\prime}=\nabla_{h}^{\prime} \Phi_{s}^{\prime}$; then the terms of order $\epsilon^{0}$ yield the unique solutions for $p^{\prime}, \theta^{\prime}, w^{\prime}$, and the velocity potential $\Phi^{\prime}$ in (10a)-(10b). Note that at this stage in the argument, the streamfunction $\Psi^{\prime}$ for the mesoscale vortical flow is not yet determined. Also, (10a) includes a familiar weak temperature gradient equation where the vertical velocity is determined by the temperature sources (see Majda and Klein 2003 and references therein).

The equatorial synoptic-scale equations are determined by averaging the three equations in (16) over the mesoscales and utilizing hydrostatic balance. The result is the equatorial synoptic-scale dynamics in (9). Note that the equations in (9) are the linear equatorial anelastic equations driven by mean sources of temperature and momentum and the vertical eddy flux divergences $-\left(\rho \overline{w^{\prime} \theta^{\prime}}\right)_{z}$ and $-\left(\rho \overline{w^{\prime} \mathbf{u}_{h}^{\prime}}\right)_{z} ;$ at this stage, the vertical eddy momentum divergence is not yet closed since it involves the vortical component of $\mathbf{u}_{h}^{\prime}$, which remains to be determined. This is done next.

First, $w_{1}^{\prime}$ is specified uniquely by the difference between the temperature equation (16b) and the average in (9b) with $\operatorname{div}_{h}^{\prime} \mathbf{u}_{h, 2}^{\prime}$ determined by the average of (16c). Recall from (5) that mesoscale derivatives involve the prime variables alone. Thus, the dynamic equation for the mesoscale fluctuating vorticity, $\omega^{\prime}\left(\mathbf{x}, \mathbf{x}^{\prime}, z, t\right)=$ $\Delta_{h}^{\prime} \Psi^{\prime}$, is determined uniquely by taking the mesocale horizontal curl of the momentum equations in (16a), that is, given $\mathbf{f}_{h}\left(\mathbf{x}, \mathbf{x}^{\prime}, z, t\right)$, curl' $\mathbf{f}_{h}=-\partial_{y^{\prime}} f_{h, 1}+\partial_{x^{\prime}} f_{h, 2}$. Using the conservation of mass equation from (10b) in (16a) yields the convenient equivalent momentum equation:

$$
\begin{aligned}
\frac{\partial \mathbf{u}_{h}}{\partial t}+\left(\mathbf{u}_{h} \cdot \nabla_{h}^{\prime}\right) \mathbf{u}_{h}+w^{\prime} \frac{\partial \mathbf{u}_{h}}{\partial z}+y \mathbf{u}_{h}^{\perp} \\
=-\nabla_{h} \bar{p}-\nabla_{h} \Phi_{s}^{\prime}-\nabla_{h}^{\prime} p_{2}+\mathbf{S}_{\mathbf{u}, 1} .
\end{aligned}
$$


The mesoscale curl' of (17) yields

$$
\begin{aligned}
\frac{\partial \omega^{\prime}}{\partial t} & +\left(\mathbf{u}_{h} \cdot \nabla_{h}^{\prime}\right) \omega^{\prime}+\left(\omega^{\prime}+y\right) \operatorname{div}_{h}^{\prime} \mathbf{u}_{h}+w^{\prime} \frac{\partial \omega^{\prime}}{\partial z} \\
& +\left(\nabla_{h}^{\prime \perp} w^{\prime}\right) \cdot \frac{\partial \mathbf{u}_{h}}{\partial z}=-\operatorname{curl}^{\prime}\left(\nabla_{h} \Phi_{s}^{\prime}\right)+\operatorname{curl}^{\prime} \mathbf{S}_{\mathbf{u}, 1} .
\end{aligned}
$$

By using (18), (10b), and $\omega^{\prime}=\Delta_{h}^{\prime} \Psi^{\prime}$, the result is the evolution equation for mesoscale vorticity listed in (10c). The derivation of BMESD is now complete. Thus, at this stage a balanced model for multiple spatial-scale and single time-scale interactions has been derived, which links the equatorial synoptic and mesoscales on the synoptic time scale. Next a more complex model is developed, which also involves temporal fluctuations on mesoscales and equatorial synoptic scales.

\section{b. Derivation of the multitime, multispace-scale MESD model}

The general multitime, multispatial-scale ansatz from (8) is substituted into the primitive equations in (3) with the assumption on the structure for the forcing in (7). The terms of order $\epsilon^{0}$ yield the linear anelastic equations in (13) for mesoscale fluctuations, and by taking the spatial average with the assumption in (7), $\partial \overline{\mathbf{u}}_{h} / \partial \tau=$ $0=\partial \bar{\theta}_{h} / \partial \tau$; the synoptic-scale variables $(\mathbf{x}, t)$ are frozen as parameters in (13). It is well known that there are explicit solution formulas for (13) with rigid-lid boundary conditions involving barotropic flow and an infinite number of constant coefficient decoupled linear shallow-water equations that can be solved by separation of variables (Gill 1982; Pedlosky 1987; Majda 2003). According to the requirement in (8), the linear forced solution in (13) should not grow secularly on the mesoscale time $\tau$ and thereby violate the low Froude number and weak temperature gradient assumptions in (4) on the longer synoptic time scale $t$. Such resonant growth will happen if and only if the forcing frequency for the appropriate component in the exact solution of (13) is proportional to the natural frequency associated with that spatial Fourier mode structure in the corresponding solution of the linear shallow-water equation (Majda 2003), that is, resonance occurs; it is not hard to explicitly classify the resonant forcings, but this is deferred to a subsequent paper involving detailed application of solutions of the present models. Here it is simply postulated that the forcing $\mathbf{S}_{\mathrm{u}}^{\prime}, S_{\theta}^{\prime}$ is nonresonant for (13). A simple illustration of the nonresonant conditions is given by the mesoscale vortical component of the linear anelastic equation in (13). The mesoscale curl' of the momentum equation in (13) yields

$$
\frac{\partial}{\partial \tau} \operatorname{curl}^{\prime} \mathbf{u}^{\prime}=\operatorname{curl}^{\prime} \mathbf{S}_{\mathbf{u}}^{\prime}
$$

Secular growth in time is avoided for a simple timedependent equation $\partial \omega^{\prime} / \partial \tau=F(\tau)$ if and only if $\langle F\rangle=0$ where $\langle\cdot\rangle$ is the mesoscale time average in (11). With this fact, secular growth for the mesoscale vorticity in (19) is avoided provided that $\operatorname{curl}^{\prime}\left\langle\mathbf{S}_{\mathbf{u}}^{\prime}\right\rangle=0$ or equivalently, $\left\langle\mathbf{S}_{\mathbf{u}}^{\prime}\right\rangle=\nabla_{h}^{\prime} \Phi_{s}^{\prime}$. This requirement is the same one derived for the single time BMESD equation in (15) by requiring the bounded leading-order terms in the primitive equations to vanish.

To derive the large-scale dynamic equations in (12) for MESD, with the ansatz in (8), the terms of order $\epsilon$ are collected, for example, in the horizontal momentum equation; they read as $\partial\left(\rho \mathbf{u}_{h, 2}\right) / \partial \tau+(16 a)$, where the notation "(16a)" is shorthand for the formula in (16a). Taking the zonal average of this results in the following equation:

$$
\frac{\partial \rho \overline{\mathbf{u}}_{h, 2}}{\partial \tau}+\overline{(16 \mathrm{a})}
$$

Now (20) has the same general form as the equation discussed below (19); the crux of the systematic mathematical argument is that one needs to avoid secular growth of $\overline{\mathbf{u}}_{h, 2}$, which would destroy the asymptotic ordering in (8); as in (19) this is automatically achieved only when the condition $\langle\overline{(16 a)}\rangle=0$ is satisfied. With the explicit terms in (16a), this is exactly the horizontal momentum equation reported in the MESD equation in (12a); a similar derivation applies for the potential temperature equation in $(12 \mathrm{~b})$ while the averaged hydrostatic balance and continuity equations in (12c)(12d) are straightforward. This completes the derivation of the multiscale MESD equations. The eddy fluxes for MESD in (12) are computed by solving (13) on the longer time scale (Majda 2003; Biello and Majda 2005, 2006a). The detailed formulas will be presented elsewhere in an explicit application.

\section{c. Comparison of the MESD and BMESD models}

The first remark here is that with the discussion below (19), the time average over mesocale fluctuations of (13) in the MESD model satisfies the same equations as the mesoscale balanced fluctuation equations for BMESD in (10). The BMESD equations, which ignore the mesoscale temporal fluctuations, systematically approximate the upscale flux of momentum and temperature from the MESD models by $\left.\overline{\left\langle w^{\prime} \mathbf{u}_{h}^{\prime}\right.}\right\rangle \approx \overline{\left\langle w^{\prime}\right\rangle\left\langle\mathbf{u}_{h}^{\prime}\right\rangle}$ and $\left\langle\overline{w^{\prime} \theta^{\prime}}\right\rangle \approx \overline{\left\langle w^{\prime}\right\rangle\left\langle\theta^{\prime}\right\rangle}$. Furthermore, in BMESD, the rotational part of $\left\langle\mathbf{u}_{h}^{\prime}\right\rangle$ is computed from the multispace single-time fluctuating vorticity equation derived in (10c). Of course the contributions in both the MESD 
and BMESD models to the turbulent fluxes on the equatorial synoptic spatiotemporal scales are both approximations that ignore all fluctuations on scales below the mesoscale, that is, below $O(150-200 \mathrm{~km})$. An extensive comparison of the two different upscale fluxes from the MESD and BMESD multiscale models will be presented in a detailed application elsewhere to wave trains of mesoscale clusters in the near future.

\section{IPESD models with multiple time scales}

Here new simplified asymptotic models are derived for equatorial synoptic- and planetary-scale waves interacting on intraseasonal time scales involving the multiple length and time scales in (2). Besides the low Froude number and weak temperature gradient assumptions in (4) for the basic flow, the source terms for momentum and temperature satisfy the multiscale assumption:

$$
\begin{aligned}
& \mathbf{S}_{\mathbf{u}}(\epsilon \mathbf{x}, \mathbf{x}, z, \epsilon t, t)=\epsilon \mathbf{S}_{\mathbf{u}, 1}^{\prime}+\epsilon^{2} \mathbf{S}_{\mathbf{u}, 2}, \\
& S_{\theta}(\epsilon \mathbf{x}, \mathbf{x}, z, \epsilon t, t)=\epsilon S_{\theta, 1}^{\prime}+\epsilon^{2} S_{\theta, 2}
\end{aligned}
$$

where $X=\epsilon x$ and $T=\epsilon t$ denote variations on the zonal planetary spatial scale and intraseasonal time scale, respectively. Also, as discussed earlier (Majda and Klein 2003; Majda and Biello 2004; Biello and Majda 2005), $\epsilon S_{\theta, 1}^{\prime}$ involves the fluctuating heating rate anomalies of $10 \mathrm{~K} \mathrm{day}^{-1}$ while $\epsilon^{2} S_{\theta, 2}^{\prime}$ involves mean heating of roughly $1 \mathrm{~K} \mathrm{day}^{-1}$. Note that for a general function $f(\mathbf{x}$, $\epsilon x, t, \epsilon t)$, by the chain rule, the zonal and time derivatives are given, respectively, by

$$
\frac{\partial f}{\partial x}+\epsilon \frac{\partial f}{\partial X}, \quad \frac{\partial f}{\partial t}+\epsilon \frac{\partial f}{\partial T}
$$

For a multiple-scale function $g(\epsilon x, x, y, z, \epsilon t, t)$, the zonal average is given by

$$
\bar{g}(X, y, z, T, t)=\lim _{L \rightarrow \infty} \frac{1}{2 L} \int_{-L}^{L} g(X, x, y, z, T, t) d x,
$$

while the temporal average is given by

$$
\langle g\rangle(X, x, y, z, T)=\lim _{\tilde{T} \rightarrow \infty} \frac{1}{2 \tilde{T}} \int_{-\tilde{T}}^{\tilde{T}} g(X, x, y, z, T, t) d t
$$

Note that $\langle\bar{g}\rangle(X, y, z, t)$ has only zonal variations on the planetary scale and temporal variations on the intrasea- sonal scale. Here and below, $g^{\prime}$ denotes a function with $\bar{g}^{\prime}=0$ as already stated in (21).

With the low Froude number and weak temperature gradient assumptions in (4), the derivation of the multiple spatiotemporal scale IPESD models begins with the ansatz (Majda and Klein 2003; Biello and Majda 2006a):

$$
u=\epsilon\left[u^{\prime}(\epsilon x, x, y, z, \epsilon t, t)+\bar{u}(\epsilon x, y, z, \epsilon t, t)\right]+\epsilon^{2} u_{2},
$$

$$
\begin{aligned}
& v=\epsilon\left(v^{\prime}+\bar{v}\right)+\epsilon^{2} \bar{v}_{2}(\epsilon x, y, z, \epsilon t, t), \\
& w=\epsilon\left(w^{\prime}+\bar{w}\right)+\epsilon^{2} \bar{w}_{2}, \\
& \theta=\epsilon\left(\theta^{\prime}+\bar{\theta}\right)+\epsilon^{2} \theta_{2}, \\
& p=\epsilon\left(p^{\prime}+\bar{p}\right)+\epsilon^{2} p_{2} .
\end{aligned}
$$

Substituting (25) into the anelastic equatorial primitive equations with (21) and (22) yields the hydrostatic balance relations $\bar{p}_{z}=\bar{\theta}, p_{z}^{\prime}=\theta^{\prime}$, as well as equations of order $\epsilon$ and order $\epsilon^{2}$ for momentum, temperature, and mass conservation. The equations of order $\epsilon$ are given by the forced linear equatorial anelastic equations for fluctuations on the equatorial synoptic scales:

$$
\begin{aligned}
\left(\rho \mathbf{u}_{h}^{\prime}\right)_{t}+y \rho \mathbf{u}_{h}^{\prime \perp} & =-\nabla_{h}\left(\rho p^{\prime}\right)+\rho \mathbf{S}_{\mathbf{u}}^{\prime}, \\
\left(\rho \theta^{\prime}\right)_{t}+\rho N^{2} w^{\prime} & =\rho S_{\theta}^{\prime}, \\
p_{z}^{\prime} & =\theta^{\prime} \\
\operatorname{div}_{h} \mathbf{u}_{h}^{\prime}+\rho^{-1}\left(\rho w^{\prime}\right)_{z} & =0
\end{aligned}
$$

and equations for the leading-order zonal averages on the synoptic time scale:

$$
\begin{aligned}
\bar{u}_{t}-y \bar{v} & =0, \\
\bar{v}_{t}+y \bar{u}+\bar{p}_{y} & =0, \\
\bar{\theta}_{t}+N^{2} \bar{w} & =0, \\
\bar{v}_{y}+\frac{1}{\rho}(\rho \bar{w})_{z} & =0 .
\end{aligned}
$$

Recall that the equatorial synoptic scale is the fast time scale compared with the intraseasonal time scale; since $\langle\partial g / \partial t\rangle=0$ for any bounded function $g$, the time average of (27) yields $\langle\bar{v}\rangle=0,\langle\bar{w}\rangle=0$, and meridional geostrophic balance,

$$
y\langle\bar{u}\rangle+\langle\bar{p}\rangle_{y}=0 .
$$

The zonal average of the terms of order $\epsilon^{2}$ for zonal momentum, potential temperature, and mass conservation are given by 


$$
\begin{aligned}
\left(\rho \bar{u}_{2}\right)_{t}+(\rho \bar{u})_{T}-\rho y \bar{v}_{2}= & -(\rho \bar{p})_{X}+\rho \bar{S}_{u, 2}-\frac{\partial}{\partial y}\left(\overline{\rho u^{\prime} v^{\prime}}\right) \\
& -\frac{\partial}{\partial z}\left(\overline{\rho u^{\prime} w^{\prime}}\right), \\
\left(\rho \bar{\theta}_{2}\right)_{t}+(\rho \bar{\theta})_{T}-\rho N^{2} \bar{w}_{2}= & \rho \bar{S}_{\theta, 2}-\frac{\partial}{\partial y}\left(\overline{\rho \theta^{\prime} v^{\prime}}\right) \\
& -\frac{\partial}{\partial z}\left(\overline{\rho \theta^{\prime} w^{\prime}}\right), \\
\bar{p}_{z}= & \bar{\theta}, \\
\frac{\partial \bar{u}}{\partial X}+\frac{\partial \bar{v}_{2}}{\partial y}+\frac{1}{\rho}\left(\rho \bar{w}_{2}\right)_{z}= & 0 .
\end{aligned}
$$

Now in order for the ansatz in (25) to remain valid, $\bar{u}_{2}, \bar{\theta}_{2}$ need to grow sublinearly in the fast time, $t$, to avoid secular growth; this requirement means that $\left\langle\partial \bar{u}_{2} / \partial t\right\rangle=0$ and $\left\langle\partial \bar{\theta}_{2} / \partial t\right\rangle=0$. Imposing this condition in (29a), (29b), recalling (28), and renaming the variables $\bar{U}=\langle\bar{u}\rangle(X, y, z, T), \bar{P}=\langle\bar{p}\rangle, \bar{\Theta}=\langle\bar{\theta}\rangle, \bar{V}=\left\langle\bar{v}_{2}\right\rangle, \bar{W}=\left\langle\bar{w}_{2}\right\rangle$, yields the planetary/intraseasonal equations:

$$
\begin{aligned}
\bar{U}_{T}-y \bar{V}+\bar{P}_{X} & =-\left\langle\overline{u^{\prime} v^{\prime}}\right\rangle_{y}-\frac{1}{\rho}\left(\rho\left\langle\overline{u^{\prime} w^{\prime}}\right\rangle\right)_{z}+\left\langle\bar{S}_{u, 2}\right\rangle, \\
y \bar{U}+\bar{P}_{y} & =0, \\
\bar{\Theta}_{T}+N^{2} \bar{W} & =-\left\langle\overline{\theta^{\prime} v^{\prime}}\right\rangle_{y}-\frac{1}{\rho}\left(\rho\left\langle\overline{\theta^{\prime} w^{\prime}}\right\rangle\right)_{z}+\left\langle\bar{S}_{\theta, 2}\right\rangle, \\
\bar{P}_{z} & =\bar{\Theta}, \\
\bar{U}_{X}+\bar{V}_{y}+\frac{1}{\rho}(\rho \bar{W})_{z} & =0 .
\end{aligned}
$$

The derivation is now complete.

These equations in (26) and (30) generalize the original IPESD models to incorporate multiple time-scale effects involving equatorial synoptic-scale temporal fluctuations. The original single time-scale IPESD models (Majda and Klein 2003; Biello and Majda 2005) are the special case of (26) and (30) where only steadystate solutions of the linear equatorial anelastic equations in (26) are utilized, and synoptic time-scale fluctuations are ignored; thus, (26) is replaced by

$$
\begin{aligned}
y \mathbf{u}_{h}^{\perp \perp} & =-\nabla_{h} p^{\prime}+\mathbf{S}_{\mathbf{u}}^{\prime}(\epsilon x, x, y, z, \epsilon t), \\
N^{2} w^{\prime} & =S_{\theta}^{\prime}(\epsilon x, x, y, z, \epsilon t), \\
p_{z}^{\prime} & =\theta^{\prime}, \\
\operatorname{div}_{h} \mathbf{u}_{h}^{\prime}+\frac{1}{\rho}\left(\rho w^{\prime}\right)_{z} & =0
\end{aligned}
$$

in the single time-scale IPESD models. Also note that the time average of (26) yields that in general, the time averages $\left\langle\mathbf{u}_{h}^{\prime}\right\rangle,\left\langle p^{\prime}\right\rangle,\left\langle\theta^{\prime}\right\rangle,\left\langle w^{\prime}\right\rangle$ also satisfy the steady-state equations in (31). One advantage of the IPESD models is their simplicity. Given the fluctuating forcing $\mathbf{S}_{\mathbf{u}}^{\prime}, S_{\theta}^{\prime}$, in (26), the planetary-scale variables $X, T$ can be regarded as frozen parameters; it is well known that there are explicit solution formulas for (26) with rigid-lid boundary conditions involving barotropic flow and an infinite number of constant coefficient decoupled linear equatorial shallow-water equations that can be solved by the separation of variables (Gill 1982; Pedlosky 1987; Majda 2003). As discussed earlier in section 3, according to the requirement in (25), the linear forced solution in (26) should not grow secularly on the synoptic time scale $t$ and thereby violate the low Froude number and weak temperature gradient assumptions on the longer intraseasonal time scale. Such resonant growth will happen if and only if the forcing frequency for the appropriate component in the exact solution of (26) is proportional to the natural frequency associated with that spatial mode in the corresponding solution of the linear equatorial shallow-water equation (Ripa 1982; Majda 2003). It is not hard to classify the resonant forcings, but this is deferred to a subsequent paper involving detailed application of solutions of the present models. Here, it is simply postulated that the forcing $\mathbf{S}_{\mathbf{u}}^{\prime}$, $S_{\theta}^{\prime}$ is nonresonant for (26). As discussed below (31), one simple necessary condition is that the time averages $\left\langle\mathbf{u}_{h}\right\rangle,\left\langle p^{\prime}\right\rangle,\left\langle\theta^{\prime}\right\rangle,\left\langle w^{\prime}\right\rangle$ satisfy the steady-state equations in (31) with source terms $\left\langle\mathbf{S}_{\mathbf{u}}^{\prime}\right\rangle,\left\langle S_{\theta}^{\prime}\right\rangle$ varying only on the intraseasonal time scale. The fluxes in (30) are computed by solving (26) on the longer time scale as for MESD (Majda 2003; Biello and Majda 2005, 2006a).

\section{An asymptotic perspective on statistical self-similarity for tropical convection}

The MESD models derived in section 3 operate on the shorter mesoscale-synoptic spatiotemporal multiple scales in (1):

$$
\left(L_{M}, L_{S}, T_{M}, T_{S}\right)=\left(\epsilon L_{S}, L_{S}, \epsilon T_{S}, T_{S}\right),
$$

while the multiple time-scale IPESD models from section 4 operate on the longer synoptic, planetary intraseasonal spatiotemporal multiscales in (2):

$$
\left(L_{S}, L_{P}, T_{S}, T_{I}\right)=\left(L_{S}, \epsilon^{-1} L_{S}, T_{S}, \epsilon^{-1} T_{S}\right) .
$$

Of course, strictly speaking, the planetary rescaling of length scales in (33) applies only for the zonal direction. Clearly, the spatiotemporal unit scales in (33) are re- 
lated to those in (32) through the rescaling transformation $\epsilon^{-1}\left(L_{M}, L_{S}, T_{M}, T_{S}\right)=\left(L_{S}, L_{P}, T_{S}, T_{I}\right)$ and the two multiscale models have substantial overlap in the equatorial synoptic spatiotemporal scales, which are the long time scales for the MESD models and the short time scales for the multitime IPESD models. See Fig. 1 for a graphical description. A natural question to ask is whether the dynamics of MESD and the multitime IPESD models are self-similar in a suitable sense (Barenblatt 2003). For a general illustrative partial differential equation with source terms, $u_{t}+f(u)_{x}=S$, under the spatiotemporal rescaling transformation $(x, t) \rightarrow$ $(\lambda x, \lambda t), u_{\lambda}(x, t)=u(\lambda x, \lambda t)$, the rescaled variable $u_{\lambda}$ satisfies

$$
\frac{\partial u_{\lambda}}{\partial t}+\frac{\partial f\left(u_{\lambda}\right)}{\partial x}=\lambda S
$$

Note that in (34) the amplitude of $u_{\lambda}$ is not rescaled, which is consistent with the low Froude number and weak temperature gradient assumptions in (4). Clearly, the spatiotemporal scaling transformation associated with the rescaling relation for the units in (32) and (33) has the value $\lambda=\epsilon$; with (34) and $\lambda=\epsilon$, the first evidence for self-similar rescaling is that the source terms in the multitime IPESD model are related to those in the MESD model by the rescaling $S_{\text {IPESD }} \approx$ $\epsilon S_{\mathrm{MESD}}$. With the structure for the source terms in MESD from (7) and that for multitime IPESD in (21), it is evident that the self-similar rescaling for the source terms is satisfied automatically. To what extent are the detailed dynamics in MESD and IPESD self-similar? In the meridional direction there is strong anisotropy due to the fact that the effects of rotation are important on the equatorial synoptic and planetary scales but are neglected to leading order on the mesoscales; however, the dynamics in the zonal direction alone along the equatorial waveguide is definitely self-similar in the following sense. Consider flows exactly along the equator, $y=0$, and ignore all $y$ fluctuations in both the multitime IPESD model in (26) and (30) and the MESD model in (12) and (13); under these special conditions both the MESD and multitime IPESD models reduce to exactly the same equations for small-scale fluctuations:

$$
\begin{aligned}
\frac{\partial u^{\prime}}{\partial \tau}+\frac{\partial p^{\prime}}{\partial x^{\prime}} & =S_{u,}^{\prime}, \\
\frac{\partial \theta^{\prime}}{\partial \tau}+N^{2} w^{\prime} & =S_{\theta}^{\prime}, \\
p_{z}^{\prime} & =\theta^{\prime}, \\
u_{x^{\prime}}^{\prime}+\frac{1}{\rho}\left(\rho w^{\prime}\right)_{z} & =0,
\end{aligned}
$$

and large-scale waves:

$$
\begin{aligned}
\frac{\partial}{\partial t}\langle\bar{u}\rangle+\frac{\partial}{\partial x}\langle\bar{p}\rangle & \left.=-\frac{1}{\rho}\left(\rho \overline{w^{\prime} u^{\prime}}\right\rangle\right)_{z}+\bar{S}_{u}, \\
\frac{\partial}{\partial t}\langle\bar{\theta}\rangle+N^{2}\langle\bar{w}\rangle & =-\frac{1}{\rho}\left(\rho\left\langle\overline{w^{\prime} \theta^{\prime}}\right\rangle\right)_{z}+\bar{S}_{\theta}, \\
\langle\bar{p}\rangle_{z} & =\langle\bar{\theta}\rangle, \\
\langle\bar{u}\rangle_{x}+\frac{1}{\rho}(\rho\langle\bar{w}\rangle)_{z} & =0
\end{aligned}
$$

in the appropriate smaller-scale variables $\left(x^{\prime}, \tau\right)$ and large-scale variables $(x, t)$ with the units in (32) and (33), respectively. Thus, there is even strong dynamic self-similarity in the zonal direction between the MESD and multitime IPESD models, even though they operate on different spatiotemporal scales.

The results derived above give the following picture for convectively coupled equatorial wave dynamics: on any fixed spatiotemporal scale from the mesoscales to the planetary scales, direct advection effects on a given scale are negligible to leading order (van Tuyl 1987); however, eddy flux divergences of momentum and temperature from nonlinear advection from the smallerscale spatiotemporal flows as well as mean source effects accumulate in time and drive the waves on the next larger scales through quasi-linear dynamics across the three fundamental spatiotemporal scales in (1) and (2) in a self-consistent fashion. This occurs in a background of mean advection provided by the large-scale flow. Furthermore, as explained above for the MESD and multitime IPESD models, these processes that transfer energy to the next larger, longer spatiotemporal scales are self-similar in a suitable sense and reflect the same physical mechanisms operating on widely different scales. Thus, the asymptotic models developed here and the analysis in this section provide evidence that strongly suggests that the observed statistical selfsimilarity among squall-line clusters, superclusters, and intraseasonal oscillations (Mapes et al. 2006) can be understood from the intrinsic self-similar dynamics in quasi-linear multiscale asymptotic models with active moisture. The present models provide strong evidence to support this hypothesis provided that the multiscale source terms from active moisture are taken to have a physically reasonable and self-consistent prescribed form. A recent multiscale study (Klein and Majda 2006) including the active nonlinear effects of moisture on scales from $10 \mathrm{~km}$ to the mesoscales $O(100 \mathrm{~km})$ on time scales of $O(20 \mathrm{~min})$ also supports the point of view developed above. Even with these smaller-scale interactions, waves on the mesoscale are driven in a quasilinear fashion through eddy momentum flux divergence 
and mean heat sources driving linear anelastic waves. Furthermore, the vertical motions on the mesoscale in this analysis are of the order $1 \mathrm{~m} \mathrm{~s}^{-1}$, which is very much compatible with the strong forcing assumptions in the MESD model on mesoscales discussed earlier.

It is worthwhile to summarize the mathematical argument presented here for self-similarity. What has been established above is that there is a simple rescaling transformation relating the shorter MESD model scales in (32) with the longer multitime IPESD scales in (33) so that as regards the dynamics in the zonal direction, the two models have identical dynamics. Furthermore, all of this is achieved through a single asymptotic parameter defined in (4) which sets the low Froude number and weak temperature gradient amplitudes as well as the rescalings between (32) and (33). Both the equations and forcing also interlock and match in this overlap so that the longtime large-scale MESD dynamic equations with forcing directly match the short time-scale IPESD model on the common overlap equatorial synoptic scales (Fig. 1). These are the key features of self-similarity through renormalization (Barenblatt 2003).

\section{Concluding discussion}

The new systematic multiscale models and methods developed in the present paper should be useful in a variety of contexts. First, as a diagnostic framework, the MESD and BMESD models should provide new insight for the fashion in which mesoscale convective clusters interact with superclusters either in observations (Wheeler and Kiladis 1999; Haertel and Kiladis 2004) or in cloud-resolving numerical models (Grabowski and Moncrieff 2001; Tulich et al. 2007); such a strategy has already proved useful in sorting out key multiscale aspects of the MJO (Majda and Biello 2004; Biello and Majda 2005, 2006a) and the new multitime IPESD models provide a framework for additional insight into these issues. An important future direction is to extend all the multiscale models in the present paper to include the active nonlinear effects of moisture across the various spatiotemporal scales considered here; the systematic multiple spatiotemporal-scale asymptotic procedures developed here combined with active nonlinear bulk microphysics or other convective parameterizations for smaller scales render this a feasible project for the near future; see Klein and Majda (2006) for systematic asymptotic models with bulk cloud microphysics for "hot towers" with mesoscale interaction from $O(10$ $\mathrm{km})$ to $O(100 \mathrm{~km})$ on the 20 -min time scale. Finally, the new MESD and BMESD models developed here that involve multispatial-scale interaction from the meso- scales on $O(150 \mathrm{~km})$ to the equatorial synoptic scales on $O(1500 \mathrm{~km})$ provide simplified models that are analogous in several respects to the "superparameterization" methods developed and applied by Grabowski (2001, 2003); another interesting future direction is to quantify the behavior of such numerical procedures through the multiple spatiotemporal-scale systematic models developed here. The author plans to explore all of these interesting issues with various collaborators in the near future.

Acknowledgments. The author warmly thanks Joseph Biello for interesting discussions on related topics. The author acknowledges generous support from the National Science Foundation under Grant NSF DMS0456713 and the Office of Naval Research under Grant ONR N00014-05-1-0164.

\section{REFERENCES}

Barenblatt, G. I., 2003: Scaling. Cambridge University Press, $\mathrm{xvi}+171 \mathrm{pp}$.

Biello, J. A., and A. J. Majda, 2005: A new multiscale model for the Madden-Julian oscillation. J. Atmos. Sci., 62, 1694-1721.

— , and — 2006a: Modulating synoptic scale convective activity and boundary layer dissipation in the IPESD models of the Madden-Julian oscillation. Dyn. Atmos. Oceans, 42, 152 215.

— , and - 2006b: Transformations for temperature flux in multiscale models for the tropics. Theor. Comput. Fluid Dyn., 20, 405-420.

Embid, P. F., and A. J. Majda, 1998: Low Froude number limiting dynamics for stably stratified flow with small or finite Rossby numbers. Geophys. Astrophys. Fluid Dyn., 87, 1-50.

Gill, A. E., 1982: Atmosphere-Ocean Dynamics. International Geophysics Series, Vol. 30, Academic Press, 662 pp.

Grabowski, W. W., 2001: Coupling cloud processes with the largescale dynamics using the cloud-resolving convection parameterization (CRCP). J. Atmos. Sci., 58, 978-997.

_ 2003 : MJO-like coherent structures: Sensitivity simulations using the cloud-resolving convection parameterization (CRCP). J. Atmos. Sci., 60, 847-864.

— tropical convection in two-dimensional explicit numerical simulations. Quart. J. Roy. Meteor. Soc., 127, 445-468.

Haertel, P. T., and G. N. Kiladis, 2004: Dynamics of 2-day equatorial waves. J. Atmos. Sci., 61, 2707-2721.

Hendon, H. H., and M. L. Salby, 1994: The life cycle of the Madden-Julian oscillation. J. Atmos. Sci., 51, 2225-2237.

Houze, R. A., 2004: Mesoscale convective systems. Rev. Geophys., 42, G4003, doi:10.1029/2004RG000150.

Kevorkian, J., and J. D. Cole, 1981: Perturbation Methods in Applied Mathematics. Applied Mathematical Sciences, Vol. 34, Springer-Verlag, $\mathrm{x}+558 \mathrm{pp}$.

Khouider, B., and A. J. Majda, 2006: A simple multicloud parameterization for convectively coupled tropical waves. Part I: Linear analysis. J. Atmos. Sci., 63, 1308-1323.

_ , and — 2007: A simple multicloud parameterization for convectively coupled tropical waves. Part II: Nonlinear simulations. J. Atmos. Sci., 64, 381-400.

Klein, R., 2000: Asymptotic analyses for atmospheric flows and 
the construction of asymptotically adaptive numerical methods. Z. Angew. Math. Mech., 80, 765-777.

__ and A. Majda, 2006: Systematic multiscale models for deep convection on mesoscales. Theor. Comput. Fluid Dyn., 20, 351-376.

Lin, J.-L., and Coauthors, 2006: Tropical intraseasonal variability in 14 IPCC AR4 climate models. Part I: Convective signals. J. Climate, 19, 2665-2690.

Majda, A. J., 2003: Introduction to PDEs and Waves for the Atmosphere and Ocean. Courant Lecture Notes in Mathematics, Vol. 9, American Mathematical Society, $\mathrm{x}+234 \mathrm{pp}$.

_, and M. G. Shefter, 2001: Models of stratiform instability and convectively coupled waves. J. Atmos. Sci., 58, 1567-1584.

— Tropics. J. Atmos. Sci., 60, 393-408.

— , and J. A. Biello, 2004: A multiscale model for the intraseasonal oscillation. Proc. Natl. Acad. Sci. USA, 101, 4736-4741.

— B. Khouider, G. N. Kiladis, K. H. Straub, and M. G. Shefter, 2004: A model for convectively coupled tropical waves: Nonlinearity, rotation, and comparison with observations. $J$. Atmos. Sci., 61, 2188-2205.

Mapes, B. E., 2000: Convective inhibition, subgrid-scale triggering energy, and stratiform instability in a toy tropical wave model. J. Atmos. Sci., 57, 1515-1535.

— , S. Tulich, J.-L. Lin, and P. Zuidema, 2006: The mesoscale convection life cycle: Building block or prototype for largescale tropical waves? Dyn. Atmos. Oceans, 42, 3-29.
Moncrieff, M. W., 2004: Analytic representation of the large-scale organization of tropical convection. J. Atmos. Sci., 61, 15211538.

_ and E. Klinker, 1997: Organized convective systems in the tropical western Pacific as a process in general circulation models: A TOGA COARE case-study. Quart. J. Roy. Meteor. Soc., 123, 805-827.

Nakazawa, T., 1988: Tropical super clusters within intraseasonal variations over the western Pacific. J. Meteor. Soc. Japan, 66, 823-839.

Pedlosky, J., 1987: Geophysical Fluid Dynamics. 2d ed. SpringerVerlag, $710 \mathrm{pp}$.

Ripa, P., 1982: Nonlinear wave-wave interactions in a one-layer reduced-gravity model on the equatorial $\beta$ plane. J. Phys. Oceanogr., 12, 97-111.

Slingo, J. M., and Coauthors, 1996: Intraseasonal oscillations in 15 atmospheric general circulation models: Results from an AMIP diagnostic subproject. Climate Dyn., 12, 325-357.

Tulich, S. N., D. Randall, and B. Mapes, 2007: Vertical-mode and cloud decomposition of large-scale convectively coupled gravity waves in a two-dimensional cloud-resolving model. $J$. Atmos. Sci., 64, 1210-1229.

van Tuyl, A. H., 1987: Nonlinearities in low-frequency equatorial waves. J. Atmos. Sci., 44, 2478-2492.

Wheeler, M., and G. N. Kiladis, 1999: Convectively coupled equatorial waves: Analysis of clouds and temperature in the wavenumber-frequency domain. J. Atmos. Sci., 56, 374-399. 